\title{
Gynaecological robotic surgery at a state hospital - our own experience
}

\author{
Berrin Göktuğ Kadioglu' ${ }^{1}$, Yakup Kumtepe ${ }^{2}$, Firdevs Sekerci Baran ${ }^{1}$ \\ ${ }^{1}$ University of Health Sciences, Erzurum Regional Traditional Hospital, Erzurum, Turkey \\ ${ }^{2}$ Atatürk University Medical Faculty, Department of Obstetrics and Gynecology, Erzurum, Turkey
}

\begin{abstract}
Objectives: In recent years, the rapid development of minimally invasive surgical methods, including robotic surgery, has resulted in a marked decline of the traditional methods in gynaecological surgery. The aim of the study was to share our experience with robotic surgery at a state hospital.

Material and methods: A total of 40 patients, who underwent robotic gynaecological surgery (GS) between 2015 and 2017, were included. Age, BMI, previous abdominal operations (PAO), operation indications (OI), operative time (OT), pathological evaluation, uterine weight (UW), blood loss during surgery (BL), complications, and duration of the hospitalization (DoH) were analyzed. The Da Vinci XI was used during surgery.

Results: A total of 40 patients were analyzed. Mean values were as follows: age -48 years, $\mathrm{BMI}-28$, and PAO $-12 \%$. The most common Ol included uterine fibroids (52\%) and abnormal uterine bleeding (45\%). Mean OT, docking time and console time values were $166 \mathrm{~min}, 15 \mathrm{~min}$, and $123 \mathrm{~min}$, respectively. Mean BL was $93 \mathrm{~mL}$. Mean UW was $256 \mathrm{gr}$, and DoH was 4 days. Perioperative and postoperative complications were observed in $10 \%$ and $20 \%$ of the cases, respectively.

Conclusions: Robotic-assisted surgery is invaluable in gynaecology, especially in the case of endometriosis, extensive adhesion, and in some oncological patients, as it allows for better visualization and higher maneuverability. In order for a surgeon to prepare for such cases, the use of the robot in benign cases is necessary to complete the learning curve and gain speed. Key words: robotic surgery, benign gynaecological disease
\end{abstract}

Ginekologia Polska 2018; 89, 9: 495-499

\section{INTRODUCTION}

Recent years have witnessed a rapid improvement of minimally invasive surgical methods, which in turn has slowly but steadily started to dethrone conventional methods in gynaecology [1, 2]. Undoubtedly, the introduction of the da Vinci robot into the field of surgery greatly contributed to that process. The first robotic hysterectomy was carried out in 2005 in the USA and ever since, da Vinci has been extensively used, especially in urologic cases [3-5].

Technically, the Da Vinci robotic system consists of 3 parts: surgeon console, 3D-HD monitoring system, and robotic arms manipulated by the surgeon. The system, due to its various advantages, is far superior to the conventional laparoscopy. A robotic system reduces physiological tremors in the hands of the surgeon to the minimum, provides
540 degrees of maneuverability by mimicking the exact movements of the surgeon's wrist, and decreases the back and neck injuries of the surgeon as a result of improved ergonomics. The disadvantages of laparoscopic surgeries include limited maneuverability as well as deteriorated display quality, and the necessity to control the equipment by hand, while the robotic camera provides perfect display quality by enlarging the image (10-20x magnification). Robotic surgery ensures ergonomic comfort and the camera is controlled by the surgeon [6]. Robot-assisted surgery has clinical advantages also for the patient, i.e. smaller incisions, less perioperative blood loss, shorter hospitalization, quicker return to the daily activities, less postoperative pain, and fewer complications $[5,7]$. Due to the fact that the manipulation rate in the robotic system is similar to the open surgery, the learning curve has

Corresponding author:

Firdevs Sekerci Baran

University of Health Sciences,

Erzurum Regional Traditional Hospital,

Erzurum, Turkey

e-mail: sekercifirdevs@gmail.com 
been observed to be shorter as compared to laparoscopy [8]. However, robotic surgery is not cost-effective as compared to the classic procedures $[9,10]$.

Hysterectomy is a common surgical solution as far as uterine complaints are concerned. In the USA, it is the second most frequent operation after C-section [9], and the most common procedure among the robotic gynaecological operations.

The aim of the study was to share our initial experiences with robotic hysterectomy.

\section{MATERIAL AND METHODS}

A total of 40 patients who underwent a robotic gynaecological operation at the /.../Maternity Hospital between 2015-2017 was analyzed. Local Ethics Committee approved of the study.

Apart from 1 subject, all patients had benign pathology and total hysterectomy was performed in all cases. The indications were similar to the usual indications for hysterectomy (Tab. 2).

Age, BMI, history of abdominal operations/C-sections, indications for surgery, operative time (first incision, port placement, docking, console time, undocking, and stitching time were included), pathological evaluation, uterine weight, blood loss during operation, complications and duration of the hospitalization were recorded.

Da Vinci XI was used in all surgeries. An identical number of ports was placed in all patients: 1 camera port, 3 ports for the arms of the robot, and 1 assistant port. The uterine manipulator was docked between the legs. The patient was operated on from the right side. A 0-degree camera and a 30-degree camera were used. Specimens were evacuated through the vaginal cuff gap, which was sutured using absorbable monofilament sutures (size 0 ) made of polyglycolic acid. The same surgical team performed all operations. Lymphadenectomy was found to be unnecessary for the one patient who was operated for endometrial carcinoma since $<50 \%$ myometrial involvement was detected.

\section{Statistical analysis}

Mean, standard deviation and percentages were evaluated. Additionally, correlations between BMI-operative time and uterine weight- operative time were investigated.

Table 1. Parameters

\begin{tabular}{|l|l|l|l|l|}
\hline & Mean & SD & n & \% \\
\hline Age [year] & 47.78 & 4.27 & 40 & \\
\hline BMI [kg/m²] & 28.18 & 4.27 & 6 & 15 \\
\hline Previous abdominal operations & & & & \\
\hline Skin-to-skin operative time [min] & 166.00 & 33.40 & & \\
\hline 'Docking' time [min] & 15.06 & 5.54 & & 10 \\
\hline 'Console' time [min] & 122.25 & 29.46 & 20 \\
\hline Complications & & & 4 & 8 \\
\hline Perioperative & & & & \\
\hline Postoperative & & & & \\
\hline Uterine weight [g] & 255.98 & 86.41 & & \\
\hline Hospitalization time [days] & 4.25 & 1.33 & & \\
\hline Perioperative bleeding volume $[\mathrm{mL}]$ & 92.75 & 122.25 & & \\
\hline
\end{tabular}

Table 2. Indications and operations

\begin{tabular}{|c|c|c|c|}
\hline Indication & n (\%) & Explanation (Accompanying pathology) & Operations \\
\hline Myoma uteri & $9(22.5)$ & & \multirow{5}{*}{$\begin{array}{l}\text { Robot-assisted hysterectomy } \\
\text { bilateral/unilateral oophorectomy } \\
\text { sub-total hysterectomy ( } 1 \text { case) } \\
\text { culdoplasty } \\
\text { Sacrouterine plication ( } 1 \text { case) } \\
\text { intestine meso-repair (1 case) } \\
\text { Adhesiolysis }\end{array}$} \\
\hline Myoma uteri+ other & $12(30)$ & $\begin{array}{l}\text { Bilateral ovarian mass, adenomyosis, HSIL, level } \\
1 \text { uterineprolapse, subserosalmyoma, pelvic pain, } \\
\text { endometrioma }\end{array}$ & \\
\hline Abnormal uterinebleeding & $5(12.5)$ & & \\
\hline $\begin{array}{l}\text { Abnormal uterine } \\
\text { bleeding + endometrial } \\
\text { pathology }\end{array}$ & $13(32.5)$ & $\begin{array}{l}\text { Chronic endometritis, endometrial polyp, endometrial } \\
\text { hyperplasia (with or without atypia), tubal metaplasia }\end{array}$ & \\
\hline Endometrialcarcinoma & $1(2.5)$ & Well-differentiated endometrioid adenocarcinoma & \\
\hline
\end{tabular}




\section{RESULTS}

In this study, data of 40 patients were retrospectively analyzed. Mean values for age and BMI were $47.78 \pm 4.27$ years (ages: 43-61) and BMI 28.18 \pm 3.76 (BMI: 23-41), respectively. The rate of previous abdominal operations was $12.5 \%$. The most common indications for surgery were uterine fibroids (52.5\%) and abnormal uterine bleeding (45\%) (Tab. 1 and 2).

Mean skin-to-skin operative time was $166 \pm 33.4 \mathrm{~min}$ (110-245 min). Mean docking and console times were $15.01 \pm 5.55 \mathrm{~min}(6-28 \mathrm{~min})$ and $122.75 \pm 29.24 \mathrm{~min}(80-$ $190 \mathrm{~min})$, respectively. In our study, we did not observe a statistically significant correlation between BMI and operative time ( $r=0.25 ; p>0.05 ;$ Fig. 1$)$. Also, no statistically significant correlations were found between uterine weight and operative time ( $r=0.33 ; p>0.05$; Fig. 2$)$.

Mean blood loss during surgery was $92.75 \pm 122.25 \mathrm{~mL}$ $(0-750 \mathrm{~mL})$. Mean uterine weight was $255.93 \pm 96.63 \mathrm{gr}$ (150-490 gr), and the mean duration of hospitalization was $4.25 \pm 1.33$ days (2-7 days) (Fig. 2 ).

Peri-operative complications were observed in 4 patients (10\%). In 1 patient, the robotic arms did not work due to the sheer size of the myoma. Hence, it was not possible to ligate the uterine artery. As the bleeding continued, conversion to laparotomy was necessary (2.5\%). In another patient, the operation was completed but, while the needle was being taken out, it dropped into the abdomen. It was found with the help of a scope but the operative time was extended. In yet another patient, perioperative hematuria was formed. It was detected by cystoscopy and repaired. Calcified bladder tumour and hematuria are formed as a result of irritation caused by the uterine manipulator. Finally, in one patient, an intestine meso-defect was formed due to trocar injury and was repaired.

Also, complications during the post-operative period were observed in 8 (20\%) patients, including 1 case of bleeding from the paravaginal artery due to insufficient cauterization during the postoperative period (the patient underwent laparotomy during the first postoperative hour), 1 case of partial intestinal obstruction in the assistant port gap on postoperative day 3 in a morbidly obese patient (the fascial defect repair was performed), and 6 cases of non-specific cardiopulmonary obstructions (tachycardia, suspicion of pulmonary embolism, chest pain, difficulty in breathing and an asthma attack).

\section{DISCUSSION}

Recent advances in robotic surgery have resulted in a wide range of possible applications in gynaecological cases $[1,2]$. A considerable learning period of the conventional laparoscopic methods and their limitations, as well as technological developments, are the reasons why more surgeons become inclined to try robotic surgery. Although the Da Vinci surgical system was first used in the USA already

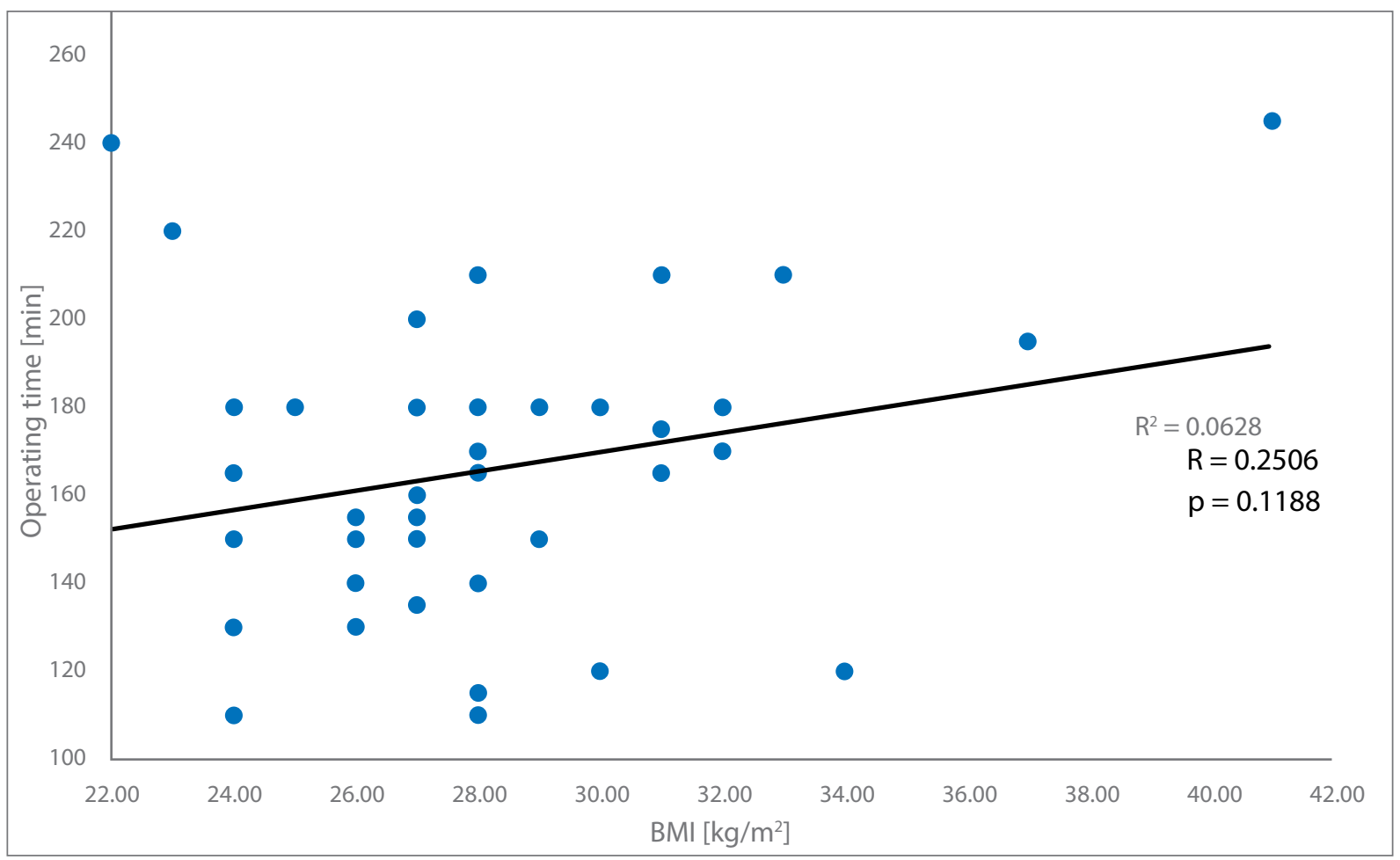

Figure 1. Correlation between BMI and operative time 


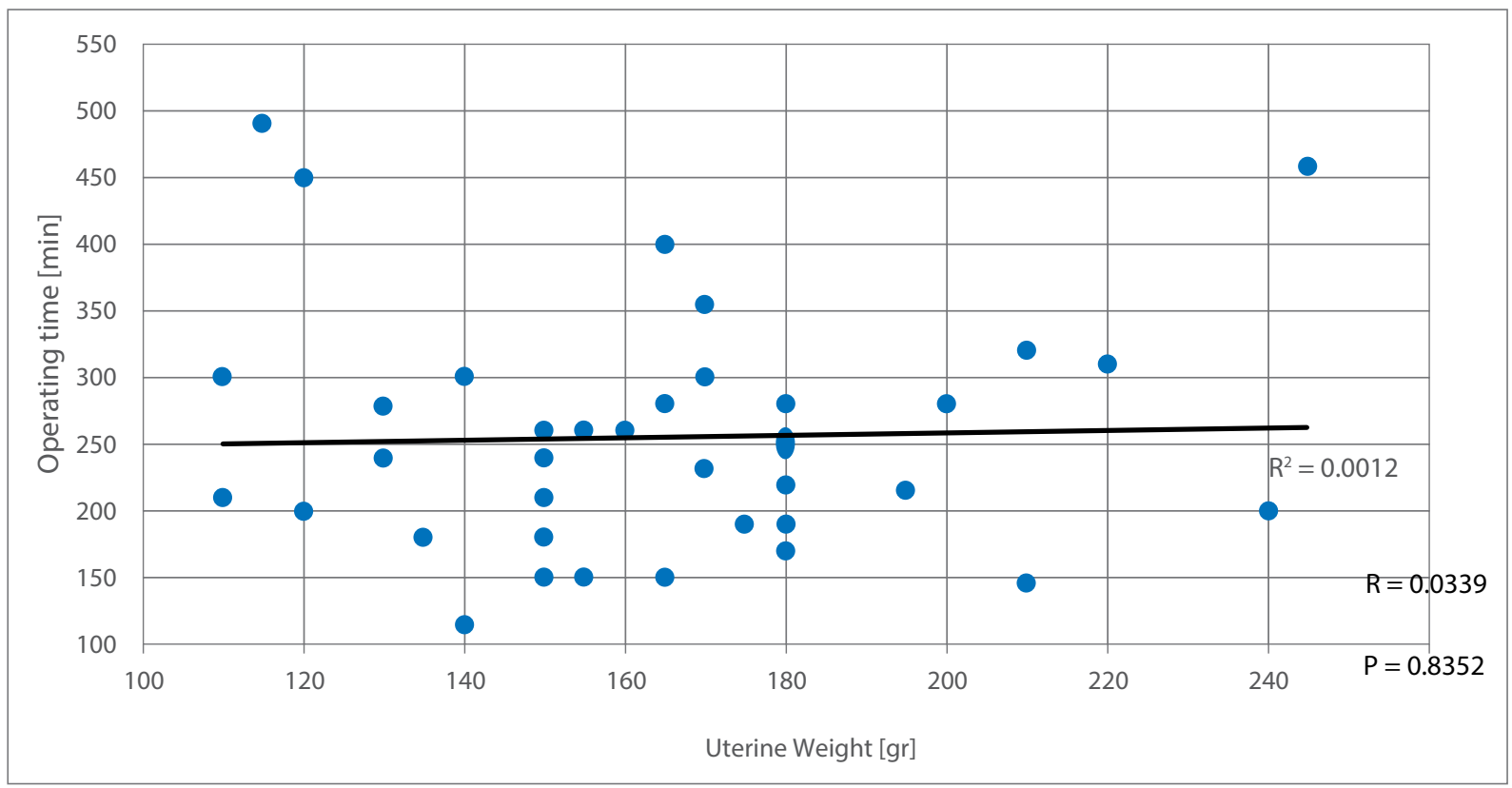

Figure 2. Correlation between uterine weight and operative time

in 1999 and was approved by the FDA in the same year, its application in the field of gynaecological surgery did not happen until 2005 [4]. The first documented usage of Da Vinci during a gynaecological operation in our country was in 2010 [11], and in our hospital in 2015.

Robotic surgery which is used for various gynaecological indications is most frequently applied in benign processes such as uterine fibroids, uterine bleedings resistant to treatment, endometrial pathologies, ovarian cysts, endometrioma, tubal pathologies, re-anastomosis, and some gynecologic oncology cases $[12,13]$.

Various studies presenting experiences with robotic surgery focus on the analysis of operative time, BMI, perioperative bleeding volume, complications and duration of hospitalization.

Operative time varies greatly between different series of robotic surgery applications. In a hysterectomy and myomectomy series of 16 patients conducted by Reynolds RK et al., in 2006, using an FDA-approved Da Vinci, mean operative time was $242 \mathrm{~min}$ [4]. Mean operative time of 75 hysterectomy cases by Goetghluck Jet al., was $128 \mathrm{~min}$ [14], and in a polycentral meta-analysis presented by Albright et al., in 162 robotic hysterectomy cases skinto-skin operative time was 108-177 min [15].

As for our study sample, composed of benign pathologies, the use of the same surgical team (primarily for hysterectomy) and the same number of ports in each patient allowed us to create a more homogeneous patient population. In light of the fact that preparation time before surgery (time before entry, anesthetization, and positioning) may vary considerably, we included the docking and undocking times and analyzed skin-to-skin operative time (which was $166 \mathrm{~min}$.) in order to keep the margin of error to the minimum. In one study, mean docking time was $4.2 \mathrm{~min}$, with the trainer involved [16]. An observer was present during three of our operations, in others, the trainer was not involved.

In our study, the average docking time was $15.06 \mathrm{~min}$. It is necessary to perform more operations in order to address the following: wide range of indications for robotic surgery, not having the same surgical team, lack of experience in the first cases, not using standard parameters, especially while calculating the operative time, and selection of the heterogeneous study population, in hopes of standardization.

The effects of BMI on the operative time were investigated by other authors, e.g. Gutierrez et al., reported a significant correlation between BMI and the operative time in robotic hysterectomies due to benign uterine diseases [16]. However, in our study, we did not observe a significant correlation between BMI and the operative time. In the same study as mentioned above, no significant correlation between uterine weight and operative time was found, which is consistent with our results.

One of the most important advantages of robotic surgery is low operative blood loss [12]. According to the literature, blood loss is considerably lower in robot-assisted surgery as compared to the conventional methods $[14,15]$. In our study, besides the cases without bleeding, mean blood loss volume was $92 \mathrm{~mL}$. The high bleeding rate in our series 
was due to the negative processes encountered in two cases. The one with the giant myoma uteri was an example of an erroneous selection and consequently, the robotic arms did not work properly so that the uterine artery was not ligatured. In the other one, approximately $200 \mathrm{~mL}$ of blood were lost due to insufficient cauterization of one of the paravaginal arteries. Laparotomy was performed in both cases. As we have mentioned, sometimes it is necessary to convert to laparotomy. The rate of these cases in the series by Goetghluck et al., with 75 robotic hysterectomy cases, was $2.7 \%$ [14]. In a meta-analysis with 326 patients that rate was $0.9 \%$ (3/326) [15], and in another polycentral analysis of 2300 cases, the rate was $0.1 \%$ [12]. In yet another study, the rate of conversion to laparotomy was higher in laparoscopic surgery as compared to robotic surgery [17].

In 2095 patients who underwent robotic hysterectomy, the most common postoperative complications were related to urinary $(1.8 \%)$ and gastro-intestinal $(0.9 \%)$ systems, while less frequent postoperative complications were cardiovascular and pulmonary ( $0.1 \%$ and $0.4 \%$, respectively) [12]. In this study, $15 \%$ of nonspecific cardiopulmonary symptoms were observed.

The cost of robotic surgery is not to be ignored. Higher costs of robotic surgery, as compared to the conventional laparoscopy, are associated with the fact that advanced technology is used $[5,10]$. The approximate cost in our study was USD 1500-2000 per case. Although this number could be considered as high, it is expected that after adaptation time the costs of the operations would be more convenient, especially in oncological and advanced endometriosis cases. Hospitalization time is another factor which affects the cost. In our study, the mean hospitalization time was 4.25 days. In contrast, in the literature, the mean hospitalization time was 1.1-3.5 days [14, 15].

Aarts et al., did not detect significant superiority of $\mathrm{RH}$ in gynaecology [18]. In a study by Simpson et al., it is underlined that there were differences regarding the opinions on the benefits of robotic surgery between the users and the literature [19]. In an article by Andres et al., robotic surgery in benign uterine diseases was not found to be superior to other classic methods [20].

\section{CONCLUSIONS}

In conclusion, robot assistance in gynaecological surgery is vital, especially in endometriosis, cases with advanced adhesion, and some oncological patients as it allows for a better display and higher maneuverability. The use of a robot in benign cases is important in order for the surgeon to complete the learning curve and gain speed, which will later be applied in the abovementioned more serious cases.

\section{REFERENCES}

1. Committee opinion no. 628: robotic surgery in gynecology. Obstet Gynecol. 2015; 125(3): 760-767, doi: 10.1097/01. AOG.0000461761.47981.07, indexed in Pubmed: 25730256.

2. Committee on Gynecologic Practice. ACOG Committee Opinion No. 444: choosing the route of hysterectomy for benign disease. Obstet Gynecol. 2009; 114(5): 1156-1158, doi: 10.1097/AOG.0b013e3181c33c72, indexed in Pubmed: 20168127.

3. Davies BL, Hibberd RD, Coptcoat MJ, et al. A surgeon robot prostatectomy-a laboratory evaluation. J Med Eng Technol. 1989; 13(6): 273-277, indexed in Pubmed: 2614807.

4. Reynolds RK, Advincula AP. Robot-assisted laparoscopic hysterectomy: technique and initial experience. Am J Surg. 2006; 191(4): 555-560, doi: 10.1016/j.amjsurg.2006.01.011, indexed in Pubmed: 16531155.

5. Wright JD, Ananth CV, Lewin SN, et al. Robotically assisted vs laparoscopic hysterectomy among women with benign gynecologic disease. JAMA. 2013; 309(7): 689-698, doi: 10.1001/jama.2013.186, indexed in Pubmed: 23423414.

6. FalconeT, Walters MD. Hysterectomy for benign disease. Obstet Gynecol. 2008; 111(3): 753-767, doi: 10.1097/AOG.0b013e318165f18c, indexed in Pubmed: 18310381.

7. Holloway RW, Patel SD, Ahmad S. Robotic surgery in gynecology. Scand J Surg. 2009; 98(2): 96-109, doi: 10.1177/145749690909800205, indexed in Pubmed: 19799047.

8. Martínez-Maestre MA, Gambadauro P, González-Cejudo C, et al. Total laparoscopic hysterectomy with and without robotic assistance: a prospective controlled study. Surg Innov. 2014; 21(3): 250-255, doi: 10.1177/1553350613492023, indexed in Pubmed: 23833240.

9. Landeen LB, Bell MC, Hubert HB, et al. Clinical and cost comparisons for hysterectomy via abdominal, standard laparoscopic, vaginal and robotassisted approaches. S D Med. 2011; 64(6): 197-199, 201, 203 passim, indexed in Pubmed: 21710804.

10. Lönnerfors $C$, Reynisson P, Persson J. A randomized trial comparing vaginal and laparoscopic hysterectomy vs robot-assisted hysterectomy. J Minim Invasive Gynecol. 2015; 22(1): 78-86, doi: 10.1016/j jmig.2014.07.010, indexed in Pubmed: 25045857.

11. Göçmen A, Sanlikan F, Uçar MG. Turkey's experience of robotic-assisted laparoscopic hysterectomy: a series of 25 consecutive cases. Arch Gynecol Obstet. 2010; 282(2): 163-171, doi: 10.1007/s00404-009-1250-6, indexed in Pubmed: 19838722.

12. Lim PC, Crane JT, English EJ, et al. Multicenter analysis comparing robotic, open, laparoscopic, and vaginal hysterectomies performed by high-volume surgeons for benign indications. Int J Gynaecol Obstet. 2016; 133(3): 359-364, doi: 10.1016/j.ijgo.2015.11.010, indexed in Pubmed: 26952352.

13. O'Sullivan OE, O'Reilly BA. Gynecological Surgery and the Robot. Engineering. 2014; 06(02): 59-70, doi: 10.4236/eng.2014.62009.

14. GoetgheluckJ, Carbonnel M, Ayoubi JM. Robotically assisted gynecologic surgery: 2-year experience in the French foch hospital. Front Surg. 2014; 1: 8, doi: 10.3389/fsurg. 2014.00008, indexed in Pubmed: 25593933.

15. Albright BB, Witte T, Tofte AN, et al. Robotic Versus Laparoscopic Hysterectomy for Benign Disease: A Systematic Review and MetaAnalysis of Randomized Trials. J Minim Invasive Gynecol. 2016; 23(1): 18-27, doi: 10.1016/j.jmig.2015.08.003, indexed in Pubmed: 26272688.

16. Gutierrez AL, Binda ML, Ramos JG. Early Experience of Robotic Hysterectomy for Treatment of Benign Uterine Disease. Rev Bras Ginecol Obstet. 2016; 38(9): 450-455, doi: 10.1055/s-0036-1592345, indexed in Pubmed: 27643518.

17. Patzkowsky KE, As-Sanie S, Smorgick N, et al. Perioperative outcomes of robotic versus laparoscopic hysterectomy for benign disease. JSLS. 2013; 17(1): 100-106, doi: 10.4293/108680812X13517013317914, indexed in Pubmed: 23743379.

18. Aarts JWM, Nieboer TE, Johnson N, et al. Surgical approach to hysterectomy for benign gynaecological disease. Cochrane Database Syst Rev. 2015(8): CD003677, doi: 10.1002/14651858.CD003677.pub5, indexed in Pubmed: 26264829.

19. Simpson KM, Advincula AP. The Essential Elements of a Robotic-Assisted Laparoscopic Hysterectomy. Obstet Gynecol Clin North Am. 2016; 43(3): 479-493, doi: 10.1016/j.ogc.2016.04.008, indexed in Pubmed: 27521880.

20. Andres Md, Borrelli GM, Abrão MS. Advances on minimally invasive approach for benign total hysterectomy: a systematic review. F1000Res. 2017; 6: 1295, doi: 10.12688/f1000research.11523.1, indexed in Pubmed: 28815020. 